\title{
Reactive Nitrogen and Oxygen Intermediates in Patients with Cutaneous Leishmaniasis
}

\section{Ozcan Erel ${ }^{+}{ }^{,}$Abdurrahim Kocyigit, Vedat Bulut*, Mehmet Salih Gurel**}

\author{
Department of Clinical Biochemistry **Department of Dermatology, Medical Faculty, Harran University, \\ Sanliurfa, Turkey *Department of Microbiology and Clinical Microbiology, Medical Faculty, \\ Firat University, Elazig, Turkey
}

The metabolisms of reactive nitrogen and oxygen intermediates (RNI and ROI) in patients with cutaneous leishmaniasis (CL) were investigated and compared with those of healthy subjects. To determine RNI metabolism, nitrite plus nitrate concentrations were measured spectrophotometrically. Nitrite concentration in plasma was determined directly by the Griess method. Nitrate levels in plasma were measured after reduction into nitrite by using copper-cadmium-zinc. ROI metabolism was evaluated by measuring erythrocyte superoxide dismutase, catalase and glutathione peroxidase activities. Plasma nitrite plus nitrate levels and erythrocyte superoxide dismutase activity were higher in the patient group than healthy subjects ( $p<0.01)$. In contrast, erythrocyte catalase and glutathione peroxidase activities were lower ( $p<0.05, p<0.01$, respectively). ROI metabolism was altered in relation to hydrogen peroxide elevation in patients with CL. These alterations in ROI enable nitric oxide (NO) to amplify its leishmanicidal effect. The determination of ROI and RNI in patients with CL may be a useful tool to evaluate effector mechanisms of $\mathrm{NO}$ and clinical manifestations.

Key words: nitric oxide - superoxide dismutase - catalase - glutathione peroxidase - leishmaniasis

Cutaneous leishmaniasis (CL) is a chronic infectious and granulomatous disease caused by Leishmania parasite and invades the skin. Parasite can multiply in macrophages. The clinical spectrum of the disease is due to the severity of the immune response of the host (Weigle \& Saravia 1996). The cellular immune response against the disease is fundamental and vitally important (Reed \& Scott 1993). Until the last decade, it was believed that free oxygen radicals were the most important part of the cellular immune response involved in killing the parasite. However, recent studies demonstrated that reactive nitrogen radicals represent the main mechanism in the elimination of parasite from the body (Liew 1992).

Studies reported hitherto have generally originated from in vitro cultures and animal experiments. Results obtained from human are very rare. In this study, the metabolisms of reactive nitrogen and oxygen intermediates (RNI and ROI) in patients with CL were investigated and compared with those of healthy subjects. In order to determine RNI metabolism, concentrations of nitrite and nitrate, oxygenated derivatives of nitric oxide (NO), were measured, whilst activities of superoxide

\footnotetext{
${ }^{+}$Corresponding author. Fax: +90-414-3151181. E-mail: gurelm@doruk.net.tr Received 23 June 1998 Accepted 4 January 1999
}

dismutase (SOD, EC 1.15.1.1), catalase (EC 1.11. 1.6) and glutathione peroxidase (GSHPx, EC 1.11.1.9) enzymes, important enzymes in ROI metabolism, in erythrocytes were determined to evaluate ROI producing or utilizing pathways. This study is an attempt to increase the knowledge concerning the immune response against intracellular parasites.

\section{MATERIALS AND METHODS}

Subjects - Patients were obtained from Leishmania Eradication Center of Sanliurfa Region which is a hyperendemic area for leishmaniasis in the southeastern Anatolia of Turkey. Subjects were 35 patients and 35 healthy individuals aged from 10-30 years from the same area. All participants gave informed consent for the present study. Subjects having secondary bacterial infection in CL lesions and other acute or chronic diseases were not included in the study.

The clinical diagnosis was confirmed by laboratory demonstration of the parasite in the lesions by direct smears. Lesions were cleaned with ethanol, and punctured at the margins of the lesion with a sterile lancet. Exudation material was smeared, dried in air and fixed by methanol. The smears were stained with Giemsa's stain for examination by light microscopy. Microscopic diagnosis was made when amastigotes were identified in the smears. In order to confirm the diagnosis, the material was also cultured in Novy MacNeal-Nicolle (NNN) 
medium for up to three weeks to detect leishmanial promastigotes. The blood samples were collected after diagnostic procedure. All the patients and the control subjects fasted for $12 \mathrm{hr}$ before their blood was collected in order to exclude dietary differences.

Measurement of plasma nitrite plus nitrate concentrations

Plasma collection - Five ml of venous blood was drawn after overnight fasting. In order to separate plasma and erythrocytes, heparinized blood was centrifuged at $1,500 \mathrm{rpm}$ for $5 \mathrm{~min}$ in normal tubes. Obtained plasma was centrifuged at 15,000 rpm for $5 \mathrm{~min}$ in eppendorf tubes. The plasma was decanted into clean tubes and stored at $-81^{\circ} \mathrm{C}$.

Catalyst for RNI assay - A catalyst to convert nitrate into nitrite were prepared as described by Davison and Woof (1978) and modified by Rockett et al. (1992). In brief, $1 \mathrm{~g}$ of powdered zinc (Merck, Germany) was washed with HCI $(1 \mathrm{~mol} / \mathrm{l})$ three times, then placed in $100 \mathrm{ml}$ of distilled water. A saturated solution of cadmium acetate (Merck) was added drop by drop. Having stringently washed with distilled water and $100 \mathrm{ml}$ of $5 \% \mathrm{CuSO}_{4}$ (Merck) slowly added onto the treated zinc, it was then washed once more with distilled water. Catalyst was stored in an $\mathrm{NH}_{4} \mathrm{Cl}$-borate buffer (Merck) adjusted to $\mathrm{pH} 8.5$, at $4^{\circ} \mathrm{C}$. The catalyst was always used within one week of preparation.

Assay for RNI - Plasma nitrite concentration was determined employing Griess reagent using the diasotization reaction as a colorimetric method (Green et al. 1982). Nitrate concentration in plasma (as well as nitrate standards) was measured after reducing it into nitrite using the catalyst, with incubation for $1 \mathrm{hr}$ at room temperature. The absorbances were read on a microplate reader (Bio-Tek Instruments, Inc., Vernont), using a test wavelength of $540 \mathrm{~nm}$ and a reference wavelength of $630 \mathrm{~nm}$.

Plasma nitrite was calculated by reading the absorbances directly from the nitrite standard curve, whereas reading plasma nitrate from the nitrate standard curve first required that the absorbance of the sample without catalyst be subtracted from the absorbance of the sample with catalyst. The results were expressed as micromolar concentrations of nitrite plus nitrate.

Estimation of activities of erythrocytic antioxidative enzymes - The buffy coat of the sediment was discarded. The remaining erythrocytes were washed repeatedly with an isotonic solution of $\mathrm{NaCl}$ until a colorless supernatant was observed. The cells were then lysed by adding four volume of cold redistilled water. The resulting suspension was centrifuged at $15,000 \mathrm{rpm}$ for $10 \mathrm{~min}$ to eliminate all of the cell membranes. Clear supernatant was obtained as hemolysate. Hemoglobin concentration of hemolysate was measured using the cyanmethemoglobin method (Von Kompen \& Zijlstra 1961). All enzyme activities were measured from the hemolysate.

Measurement of erythrocyte SOD and GSHPX activities - Erythrocyte superoxide dismutase (SOD) and glutathione peroxidase (GSHPx) activities were determined using haemolysates and commercially available kits (Randox Lab. Ltd, Ireland Cat.No. SDI 25 and RS505 respectively).

SOD activity estimation was based on the generation of superoxide radicals produced by xanthine and xanthine oxidase, which reacts with 2(4-iodophenyl)-3-(4-nitrophenol)-5phenyltetrazolium chloride to form a red formazan dye (Mccord \& Fridowich 1969). The SOD activity is then measured by the degree of inhibition of this reaction. Erythrocyte SOD activity was expressed as U/g Hb.

GSHPx activity estimation is based on the following principle: GSHPx catalyses the oxidation of glutathione by cumen hydroperoxide (Paglia \& Valentine 1967). In the presence of glutathione reductase and nicotinamide adenine dinucleotide phosphate (NADPH) the oxidized glutathione is immediately converted to the reduced form with a concomitant oxidation of NADPH to NADP ${ }^{+}$. The decrease in absorbance at $340 \mathrm{~nm}$ is measured. Erythrocyte GSHPx activity was expressed as U/g $\mathrm{Hb}$.

Measurement of erythrocyte catalase activity Erythrocyte catalase activity was assayed according to the modified technique of Hagglof et al. (1983) by the following the absorbance of hydrogen peroxide at $240 \mathrm{~nm}$ using a spectrophotometer (Cecil 2000, England). One unit of catalase activity was defined as the amount of enzyme that composes $1 \mathrm{mmol} \mathrm{H}_{2} \mathrm{O}_{2} / \mathrm{min}$ at an initial concentration of $10 \mathrm{mmol} / \mathrm{l}$ at $\mathrm{pH} 7.4$ and $25^{\circ} \mathrm{C}$. A standard curve was constructed by using purified bovine liver catalase (Sigma). Erythrocyte catalase activity was expressed as $\mathrm{kU} / \mathrm{g} \mathrm{Hb}$.

Statistics - Statistical analyses were performed by using SPSS for windows version 6.0 computer program. The mean values of the groups were compared with Student's $t$ test. All results were expressed as mean \pm one $\mathrm{SD}$, and significance was defined as $\mathrm{p}<0.05$.

\section{RESULTS}

The patients and controls were similar in age, height, body weight and body mass index. The mean duration of the disease was $5.5 \pm 3.3$ months and the mean area of lesions was $3.4 \pm 2.9 \mathrm{~cm}^{2}$. As shown in the Table, plasma nitrite plus nitrate concentrations and erythrocyte SOD activity were 
TABLE

Plasma nitrite plus nitrate concentrations, erythrocyte superoxide dismutase, catalase and glutathione peroxidase activities in patients with cutaneous leishmaniasis and healthy subjects

\begin{tabular}{lccc}
\hline & Patients group & Healthy subjects & $p^{a}$ \\
\hline Nitrite plus nitrate, $\mathrm{mmol} / \mathrm{l}$ & $76.3 \pm 32.5$ & $28.1 \pm 26.8$ & $<0.01$ \\
Superoxide dismutase, $\mathrm{U} / \mathrm{g} \mathrm{Hb}$ & $609.2 \pm 75.4$ & $456.6 \pm 67.3$ & $<0.01$ \\
Catalase, $\mathrm{kU} / \mathrm{g} \mathrm{Hb}$ & $119.6 \pm 20.5$ & $135.1 \pm 21.7$ & $<0.05$ \\
Glutathione peroxidase, $\mathrm{U} / \mathrm{g} \mathrm{Hb}$ & $313.5 \pm 65.2$ & $398.4 \pm 68.3$ & $<0.01$ \\
\hline
\end{tabular}

Values are mean $\pm \mathrm{SD} ; a$ : values less than 0.05 are significant obtained from Student's $t$ test.

found to be higher in CL patients when compared to healthy individuals, while erythrocyte catalase and GSHPx activities were found to be lower in the patient group than in the control group.

\section{DISCUSSION}

Nitric oxide, a free radical, (NO*) is an important mediator of both physiological and pathophysiological processes (Moncada et al. 1991). NO is produced in a pathway catalyzed by nitric oxide synthase (NOS; EC 1. 14.13.39), an enzyme that exists in three isoforms encoded by distinct genes (Knowles \& Moncada 1994). All isoforms of NOS catalyse the conversion of L-arginin into citrulline and NO.

Neuronal NOS (type I, nNOS) and endothelial NOS (type III, eNOS) have physiological steady state activity. Inducible NOS (type II, iNOS) is not expressed under normal conditions. iNOS is induced by cytokines and endotoxins during inflammatory and infectious processes and produces huge amounts of NO for extended periods (Erbas 1997). Macrophages, neutrophils and mast cells have all been shown to be major producers of this molecule (Marletta et al. 1988). NO produced by iNOS has an antimicrobial activity and may be involved in killing tumor cells. As such, it is part of the nonspecific host defense system. Increased expression of iNOS has been demonstrated in a wide range of disorders, including sepsis, asthma, rheumatoid arthritis, atherosclerotic lesions, tuberculosis, inflammatory bowel disease, Helicobacter pylori induced gastritis, allograft rejection, Alzheimer disease, and multiple sclerosis (Moshage 1997). However, there is no report concerning patients with CL. Previous studies related to nitric oxide production in leishmaniasis have used either macrophage culture or animal models. In patients with CL, we determined that plasma nitrite plus nitrate concentrations are higher than those of healthy subjects.

CL has been continuing endemically for a long time in Sanliurfa, a city in Southeastern Anatolia of Turkey, which is the largest focus of CL in the county. The leishmanial strains described by Gramiccia et al. (1984) were Leishmania tropica which agrees with the clinical observations. Five hundred and fifty and 4,000 cases of CL were reported by health ministry division of Sanliurfa in 1990 and 1994 respectively (Desjeux 1997).

NO has a very short half-life. Within seconds of being produced, it is converted to nitrite and nitrate, sequentially, in the presence of molecular oxygen. Therefore, measurements of nitrite and nitrate in plasma indicate the production of NO (Archer 1993). Since the half-life of nitrate in plasma is approximately $1.5 \mathrm{hr}$, nitrate concentration may be an end-point parameter for NO production (Moshage 1997). These molecules, including $\mathrm{NO}$ and its oxidized forms, nitrite $\left(\mathrm{NO}_{2}^{-}\right)$and nitrate $\left(\mathrm{NO}_{3}{ }^{-}\right)$are termed RNI.

Previous experimental studies have shown that interferon- $g$ (IFN-g), tumor necrosis factor (TNF), interleukin-1 (IL-1), interleukin-2 (IL-2) and lipopolysaccharides (LPS) induce iNOS enzyme and lead to the elevated nitric oxide synthesis (Sessa 1994). Furthermore, it was reported that IFN-g, TNF and IL-1 levels were increased in the sera of patients with cutaneous leishmaniasis (Liew \& O’Donnell 1993).

Determination of plasma nitrite plus nitrate concentrations mainly reflects NO produced by the activity of iNOS, since, NO produced by means of eNOS and nNOS is nearly a thousand times lower (Archer 1993).

The mechanism by which NO kills cells and parasite has yet to be clarified. NO can covalently react with intracellular iron, thus reacting with Fe-S prosthetic groups of susceptible enzymes, e.g. aconitase and complex I and II of the mitochondrial electron transport chain. This results in the formation of iron-nitrosyl complexes, the inactivation and degradation of these enzymes and the cessation of DNA replication (Liew \& O'Donnell 1993). It has been confirmed by several laboratories that control of leishmania infection in the murine model is NO dependent. Leishmaniacidal ac- 
tivity in vitro is dramatically reduced by the arginine analogue L-NG-monomethyl-arginine (LNMMA), which is a NOS competitive inhibitor (Mcsorley et al. 1996).

On the other hand, macrophages when exposed to microorganisms or a chemotactic stimulus, exhibit a burst in oxygen consumption coincident with the generation of ROI, such as superoxide, hydrogen peroxide, through the activation of NADH oxidase (Ockenhouse \& Shear 1984).

Unlike to RNI metabolites, ROI metabolism in macrophages can not be evaluated by means of plasma values. Additionally, there are already some studies to investigate the changes in RNI and ROI metabolisms in macrophages stimulated with antigens and certain cytokines (Liew \& O'Donnell 1993). Immune mediators produced by the host against Leishmania as defense mechanisms effect the metabolisms of RNI and ROI. Owing to the fact that the effects of cytokines in the circulation is systemic, other cells are likely to be affected. Erythrocytes abundantly contain SOD, catalase and GSHPx enzymes, and they reflect alterations in the activities of these enzymes well. Therefore, we evaluated ROI metabolism changes in CL by determining the activities of these enzymes in erythrocytes.

In this study, we found that plasma nitrite plus nitrate levels and erythrocyte SOD activity were higher in the patient group than those of healthy subjects, and erythrocyte catalase and GSHPx activities were decreased. Gryglewski et al. (1986) demonstrated that superoxide anion diminished the effects of nitric oxide and the addition of SOD into the media negated the negative effect of superoxide anion. Li et al. (1992) reported that SOD enzyme increased and catalase enzyme decreased the leishmanicidal effect of NO, in vitro. Ding et al. (1988) reported that IFN-gled to the elevated production of nitrite and hydrogen peroxide via two independent pathways. Our report is the first report demonstrating erythrocyte SOD, catalase and GSHPx activities in relation to CL.

SOD converts superoxide radical into hydrogen peroxide. Catalase and GSHPx enzymes utilize hydrogen peroxide as substrate. GSHPx has lower a $\mathrm{K}_{\mathrm{m}}$ and higher affinity for $\mathrm{H}_{2} \mathrm{O}_{2}$ than catalase has. Increases in SOD activity result in the convertion of superoxide anion, which has a negative effect of the effects of NO, into hydrogen peroxide. On the other hand, decreases in GSHPx and catalase activities enable hydrogen peroxide to stay in the medium for a long time and at high concentrations. Rockett et al. (1991) in their in vitro study, demonstrated that NO derivatives, such as nitrite, nitrate, peroxynitrite, were more toxic against Plasmodium falciparum parasite than NO itself. Swain et al. (1994) obtained in vitro in a simple experiment peroxynitrite, a very toxic agent, by just mixing nitrite, nitrate and hydrogen peroxide.

It is easily predicted that ROI metabolism changes, such as increased levels of hydrogen peroxides, as we observed in the erythrocytes of leishmaniasis patients are most likely to be found in macrophages, as well. In induced macrophages, NO produced in huge amounts, with hydrogen peroxides, shows more potent leishmaniacidal effect by being converted into more toxic derivatives, such as peroxynitrite. In in vivo and in vitro studies performed to date, it has been indicated that TNF only induces SOD activity, but IFN-gstimulates both SOD and iNOS activities.

Consequently, RNI metabolism is altered in patients with CL, and, on the other hand, ROI metabolism is regulated to amplify the leishmaniacidal effect of RNIs. In CL patients, assessments of RNI and ROI metabolisms may be useful tools in the evaluation of the effector mechanisms of macrophages and clinical manifestations of patients.

\section{REFERENCES}

Archer S 1993. Measurement of nitric oxide in biological models. FASEB J 7: 349-360.

Davison W, Woof C 1978. Comparison of different forms of cadmium as reducing agent for the batch determination of nitrate. Analyst (London)162: 403-408.

Desjeux P 1997. Leishmaniasis. Public health aspects and control. Clin Dermatol 14: 417-423.

Ding AH, Nathan CF, Stuehr D 1988. Release of reactive nitrogen intermediates and reactive oxygen intermediates from mouse peritoneal macrophages. Comparison of activating cytokines and evidence for independent production. J Immunol 141: 2407-2412.

Erbas D 1997. Nitric oxide in health. Turk J Med Sci 27 : 501-507.

Gramiccia M, Bettini S, Yasarol S 1984. Isoenzyme characterization of Leishmania isolates from human cases of cutaneous leishmaniasis in Urfa, southeast Turkey. Trans R Soc Trop Med Hyg 78: 568.

Green LC, Wagner DA, Glogowski J, Skipper PC, Wishnok JS, Tannenbaum SR 1982. Analyses of nitrate, nitrite and $\left[{ }^{15} \mathrm{~N}\right]$ nitrite in biological fluids. Anal Biochem 126: 131-138.

Gryglewski RJ, Palmer RM, Moncada S 1986. Superoxide anion is involved in the breakdown of endothelium-derived vascular relaxing factor. Nature 320 : 454-456.

Hagglof B, Marklund SL, Holmgren G 1983. CuZn superoxide dismutase, $\mathrm{Mn}$ superoxide dismutase, catalase and glutathione peroxidase in lymphocytes and erythrocytes in insulin dependent diabetic children. Acta Endocrinol 102: 235-239.

Knowles RG, Moncada S 1994. Nitric oxide syntheses in mammals. Biochem J 298: 249-258.

Li Y, Severn A, Rogers MV, Palmer RM, Moncada S, Liew FY 1992. Catalase inhibits nitric oxide synthesis and the killing of intracellular Leishmania 
major in murine macrophages. Eur J Immunol 22: 441-446.

Liew FY 1992. Induction, regulation and function of Tcell subsets in leishmaniasis. Chem Immunol 54: 117135.

Liew FY, O’Donnell CA 1993. Immunology of leishmaniasis. Adv Parasitol 32: 162-222.

Marletta MA, Yoon PS, Lyengar R, Leaf CD, Wishnok JS 1988. Macrophage oxidation of L-arginine to nitrite and nitrate: nitric oxide is an intermediate. Biochemistry 27: 8706-8711.

McCord JM, Fridowich I 1969. Superoxide dismutase. An enzymatic function for erythrocuprein (hemocuprein). J Biol Chem 244: 6049-6055.

McSorley S, Proudfoot L, O’Donnell CA, Liew FY 1996. Immunology of murine leishmaniasis. Clin Dermatol 14: 451-464.

Moncada S, Palmer RM, Higgs EA 1991. Nitric oxide: physiology, pathophysiology, and pharmacology. Pharmacol Rev 43: 109-142.

Moshage H 1997. Nitric oxide determinations; much a do about NO* -thing? Clin Chem 43: 553-557.

Ockenhouse CF, Shear HL 1984. Oxidative killing of the intraerythrocytic malaria parasite Plasmodium yoelii by activated macrophages. J Immunol 132: 424-431.
Paglia DE, Valentine WN 1967. Studies on the quantitative and qualitative characterization of erythrocyte glutathione peroxidase. J Lab Clin Med 70: 158-159.

Reed SG, Scott P 1993. T-cell and cytokine responses in leishmaniasis. Curr Opin Immunol 5: 524-531.

Rockett KA, Awbum NW, Aggarwal BB, Cowden WB, Clark IA 1992. In vivo induction of nitrite and nitrate by tumor necrosis factor, lymphotoxin and interleukin-1: possible roles in malaria. Infect Immun 60: 3725-3730.

Rockett KA, Awburn MM, Cowden WB, Clark IA 1991. Killing of Plasmodium falciparum in vitro by nitric oxide derivatives. Infect Immun 59: 3280-3283.

Sessa WC 1994. The nitric oxide synthase family of proteins. J Vasc Res 31: 131-143.

Swain JA, Darley-Usmer V, Gutteridge JM 1994. Peroxynitrite releases copper from caeruloplasmin: implications for atherosclerosis. FEBS Lett 342: 4952.

Von Kompen EJ, Zijlstra WG 1961. Standardization of hemoglobinometry. II. The hemoglobincyanide method. Clin Chem Acta 6: 538-544.

Weigle K, Saravia NG 1996. Natural history, clinical evolution, and the host-parasite interaction in new world cutaneous leishmanisis. Clin Dermatol 14: 433-450. 
Free Radical Metabolisms in Cutaneous Leishmaniasis • Ozcan Erel et al. 